\title{
Estimating the Prevalence of Pseudomyxoma Peritonei in Europe Using a Novel Statistical Method
}

\author{
Thale Dawn J. H. Patrick-Brown, MSc ${ }^{1,2}$, Norman John Carr, MD, $\mathrm{PhD}^{3}$ (ㄷ), David M. Swanson, $\mathrm{PhD}^{4}$, \\ Stein Larsen, MD, $\mathbf{P h D}^{5}$, Faheez Mohamed, $\mathrm{MD}, \mathbf{P h D}^{3}$, and Kjersti Flatmark, MD, PhD ${ }^{1,5,6}$ \\ ${ }^{1}$ Department of Tumor Biology, The Norwegian Radium Hospital, Oslo University Hospital Oslo, Oslo, Norway; \\ ${ }^{2}$ Department of Oncology, Akershus University Hospital, Lørenskog, Norway; ${ }^{3}$ Peritoneal Malignancy Institute, \\ Basingstoke and North Hampshire Hospital, Aldermaston Road, Basingstoke, UK; ${ }^{4}$ Oslo Centre for Biostatistics and \\ Epidemiology (OCBE), University of Oslo, Oslo, Norway; ${ }^{5}$ Department of Tumor Biology, Institute for Cancer Research, \\ The Norwegian Radium Hospital, Oslo University Hospital, Oslo, Norway; ${ }^{6} \mathrm{Faculty}$ of Medicine, University of Oslo, Oslo, \\ Norway
}

\begin{abstract}
Background. The determination of the incidence and prevalence of rare diseases is important for economists and health-care providers. Pseudomyxoma peritonei (PMP) is a rare, slow-growing abdominal cancer that represents a substantial burden on both patients and health-care systems. The incidence rate was previously approximated at $1-2$ people per million per year; this incidence has never been challenged, and the prevalence has not been estimated.

Methods. Epidemiological data from Norway and England were obtained and analysed to calculate a minimum incidence rate based on the number of patients having a first surgical intervention for PMP. A novel method was then used to determine a prevalence rate for PMP, incorporating incidence, death, and cure rates in a multi-year analysis that accounted for the increasing population of Europe over a 10 -year period.

Results. An incidence rate of 3.2 people per million per year was calculated, with a corresponding estimated prevalence rate of 22 people per million per year. By this calculation, 11,736 people in Europe were estimated to be living with PMP in 2018.
\end{abstract}

Conclusion. Incidence and prevalence are essential tools for assessment of the financial and human cost of a disease.

(C) The Author(s) 2020

First Received: 19 March 2020;

Published Online: 2 June 2020

K. Flatmark, MD, PhD

e-mail: kjersti.flatmark@rr-research.no
For rare diseases, such as PMP, the lack of accurate registries presents a particular challenge in determining such health-related statistical parameters. Based on our calculations, a significant number of people are living with PMP in Europe, underlining the need for appropriate resource allocation to ensure that adequate health-care measures are provided.

The estimation of the number of people suffering from rare diseases is important to the health-care community. Statistical indicators help shed light on not only the number of new cases that may present each year (incidence), but also on the number of people who might be affected by the disease at any given time (prevalence). These figures assist clinicians, medical economists and politicians with long-term planning for the use of resources and infrastructure, such as bed and facility planning, staffing, and budgeting. Monitoring of incidence and prevalence is also useful for prevention and treatment strategies, as well as understanding the continuing human cost of the disease. Underestimating incidence and prevalence leads to inadequate provision of support to patients who may require repeated surgical interventions and ongoing medical treatment. This further impacts the economy through people's inability to work or take an active role in the community due to symptoms and disability.

The monitoring of health-related statistics through national databases lends itself to the accurate calculation of incidence and prevalence, allowing good quality data to be produced for forecasting and planning activities. Due to lack of resources, the demographics of rare diseases can be 
challenging to determine using standard medical reporting tools, making useful predictive models difficult to establish. Less common diseases may further suffer from lower rates of reporting, under-diagnosis or misdiagnosis, leading to underestimation of both incidence and prevalence.

Pseudomyxoma peritonei (PMP) often called "jellybelly", is a rare cancer that generally presents as multifocal mucinous tumours in the abdominal cavity causing increased abdominal girth, pain and pressure on internal organs due to the accumulation of large amounts of mucinous tumour. PMP most commonly originates from tumours of the appendix that rupture, seeding cancerous cells throughout the abdominal cavity via paths of fluid reabsorption. Treatment involves cytoreductive surgery (CRS) and hyperthermic intraperitoneal chemotherapy (HIPEC) which, when performed at specialist centres, can lead to an overall 10-year survival of around $63 \% .^{1}$ For patients that cannot be cured by surgery, no effective treatments exist, and since PMP is a slow-growing cancer, patients may live for many years with active disease and worsening symptoms, presenting a substantial burden on the health-care system. Estimation of prevalence is therefore essential for resource allocation.

Prevalence estimates depend on the incidence rate, which for PMP have been widely quoted as approximately 1-2 people per million. ${ }^{2}$ This estimation was made in a study looking at neoplasms of known appendiceal origin and included only the low-grade subtype of PMP, so some cases may have been left out of the calculation. It is widely believed by experts in the field that the total number of incident cases is higher because PMP is often misdiagnosed, leading to an under-reporting of the condition. As formal registries for PMP do not exist, challenges in extrapolating figures have meant that incidence and prevalence have been difficult to calculate.

This paper seeks to determine a scientifically grounded incidence rate based on the number of cases having surgical intervention in one country (Norway) over a 10-year period, validated by data from England. The paper also outlines a novel waterfall method for calculating prevalence in an increasing population and then presents an estimated prevalence of PMP for the European population.

\section{MATERIALS AND METHODS}

\section{Patients}

To determine the incidence rate of PMP in Norway, data from records of patients treated for PMP between 2009 and 2018 at the Norwegian Radium Hospital, part of Oslo University Hospital Comprehensive Cancer Centre, were obtained. The Norwegian Radium Hospital has performed
CRS and HIPEC since 1994, and serves the entire Norwegian population of more than five million through the "Norwegian National Unit for Hyperthermic Intraperitoneal Chemotherapy in Colorectal Cancer, Pseudomyxoma Peritonei and Abdominal Mesothelioma". Data for England included patients treated for PMP at the Peritoneal Malignancy Institute, Basingstoke, between 2012 and 2018, who lived in the UK regions of London, the South East, and South West of England, East and West Midlands, Wales, Guernsey, Jersey and the East of England. All PMP cases were verified by an expert pathologist.

\section{Population Data}

European population statistics for the years 1989-2018 were obtained from the United Nations' Department of Economic and Social Affairs Population Division, ${ }^{3}$ which provides population estimates using various time-tested methods, described in their manual. ${ }^{4}$ Overall population numbers were identified for Austria, Belgium, Bulgaria, Croatia, Cyprus, Czech Republic, Denmark, Estonia, Finland, France, Germany, Greece, Hungary, Ireland, Italy, Latvia, Lithuania, Luxembourg, Malta, the Netherlands, Norway, Poland, Portugal, Romania, Slovakia, Slovenia, Spain, Sweden and the United Kingdom (hereafter referred to as Europe). Annual population estimates for administrative regions within England were obtained from reports produced by the UK Office of National Statistics. ${ }^{5}$

\section{Surveillance Period}

To determine the appropriate length of time to consider a patient under active surveillance (with or without active disease), articles reporting survival parameters after surgery for PMP were consulted. Published PMP survival curves appear to plateau by year 10 , and almost all recurrences $(98.7 \%)$ are reported to have occurred by that time. ${ }^{6-16}$ A surveillance period of 10 years was therefore adopted to calculate the period prevalence.

\section{Annual Death Rates}

Due to the length of the period covered and the slow growth rate of PMP, the model required a reductive technique that decreased patient numbers in line with known survival rates. The prevalence calculation method presented here used estimated annual death rates over the 10-year surveillance period to remove non-surviving cases from the cohort each year. Curves reporting overall survival (OS) reflect the total number of patients under surveillance, regardless of disease status. Published OS curves for PMP are relatively uniform, and for a representative estimation of annual death rates, one of the 
largest PMP cohorts reported was used. ${ }^{1}$ The proportion of patients lost from the cohort each year was manually estimated from the survival curve and the resulting percentages of deceased (cumulative) are presented in Table 1.

\section{Cure Rate}

Cases that had not experienced disease recurrence at year 10 were considered cured and removed from the calculation, and the reported 10-year progression-free survival (PFS) was therefore considered the cure rate. Reported PFS varies according to histological subtype, ranging from 32 to $86 \%$ in high- and low-grade subtypes, respectively, ${ }^{1,16}$ with low-grade disease being the most commonly diagnosed. In studies where all histological subtypes were included, the low-grade sub-type usually constituted around $70 \%$ of cases presenting for surgery. ${ }^{1,7,17}$ Ten-year PFS of between $47 \%$ and $54 \%$ was reported for the populations identified within the studies. ${ }^{1,8,14,18}$ For the purpose of the prevalence calculation in this paper, a conservative cure rate of $47 \%$ at year 10 was adopted.

\section{RESULTS}

\section{PMP Incidence Rates in Norway}

Based on the statistics obtained from the Norwegian Radium Hospital, the incidence of PMP in Norway was calculated for the years 2009-2018 based on the date of first surgical intervention (Table 2). The number of new people with PMP each year was between 10 and 25, which gave an incidence range of between 2 and 5 per million, with a mean incidence rate of 3.2 people per million per year over the period. The incidence rate of 3.2 people per million was therefore adopted as the base incidence rate used for calculations of prevalence rate.

TABLE 1 Death rate from pseudomyxoma peritonei, percentage of total patient cohort deceased per year

\section{PMP Incidence Rates in England}

The Peritoneal Malignancy Institute (Basingstoke) treated between 119 and 160 patients from the selected areas per annum between 2012 and 2017, with an annual average of 138 patients. The incidence rate was calculated using the same method as for the Norwegian cohort. The mean incidence rate for England mirrored that in Norway, with 3.2 people per million per annum (Table 2).

\section{European PMP Incidence}

Based on the incidence rate obtained from the Norwegian and British populations, an estimation of the incidence of PMP in Europe was calculated. Table 3 shows the calculated incidence between the years 2009 and 2018 for the European population. In 2018, the estimated incidence for Europe was 1696 people.

\section{The Waterfall Prevalence Calculation}

Using the incidence rate of 3.2 people per million, a cure rate of $47 \%$, and the death rates shown in Table 1, the prevalence of PMP in Europe was determined using a novel waterfall method designed to capture the overall number of patients living with the disease. The number of patients in the primary cohort each year ("total annual cohort") was determined using the calculated European incidence (Table 3). The cohort was decayed each year according to the figures noted in Table 1 , and at year 10, the $47 \%$ were removed from the overall calculation, reflecting the cure rate. Table 4 shows how the waterfall method was applied to determine the prevalence from 2008 to 2018. "Total period cohort" refers to the accumulation of the annual incident cohorts over a 10 -year period, i.e. all cases newly diagnosed (Year 1) or continuing to live with the disease each year (Years 2-10) over the decade.

The actual calculations were initiated in the year 1998; the 10-year time-span between 2009 and 2018 is shown in Table 4 . To determine the period prevalence, average total cohorts minus 10 -year survivors across the 10 -year period were calculated, and the average resulting cohort size was divided by the average European population, giving an overall prevalence rate of 22 people per million, or a total of 11,736 people living with PMP in Europe in 2018.

\section{DISCUSSION}

In this paper, we propose a novel method for period prevalence calculation applied to PMP, which is a slowgrowing rare cancer. The method allows a more accurate estimate for diseases where a small difference in incidence 
TABLE 2 PMP incidence rates in Norway and England

\begin{tabular}{|c|c|c|c|c|c|c|}
\hline \multirow[t]{2}{*}{ Year } & \multicolumn{3}{|c|}{ Norwegian population data } & \multicolumn{3}{|c|}{ English population data } \\
\hline & Population & Number of PMP cases & Incidence rate & Population & Number of PMP cases & Incidence rate \\
\hline 2009 & $4,779,252$ & 10 & 2.1 & & & \\
\hline 2010 & $4,858,199$ & 16 & 3.3 & & & \\
\hline 2011 & $4,920,305$ & 10 & 2.0 & & & \\
\hline 2012 & $4,985,870$ & 14 & 2.8 & $41,726,543$ & 127 & 3.0 \\
\hline 2013 & $5,051,275$ & 17 & 3.4 & $42,059,410$ & 119 & 2.8 \\
\hline 2014 & $5,109,056$ & 18 & 3.5 & $42,460,537$ & 160 & 3.8 \\
\hline 2015 & $5,165,802$ & 16 & 3.1 & $42,861,652$ & 134 & 3.1 \\
\hline 2016 & $5,213,985$ & 14 & 2.7 & $43,265,798$ & 139 & 3.2 \\
\hline 2017 & $5,258,317$ & 25 & 4.8 & $43,559,112$ & 150 & 3.4 \\
\hline 2018 & $5,337,962$ & 20 & 3.8 & & & \\
\hline
\end{tabular}

PMP pseudomyxoma peritonei

TABLE 3 Estimated number of new cases of pseudomyxoma peritonei per year across Europe from 2009 to 2018

\begin{tabular}{lll}
\hline Year & Total European population & Incidence \\
\hline 2009 & $518,299,528$ & 1659 \\
2010 & $519,963,424$ & 1664 \\
2011 & $521,489,644$ & 1669 \\
2012 & $522,872,037$ & 1673 \\
2013 & $524,147,779$ & 1677 \\
2014 & $525,373,520$ & 1681 \\
2015 & $526,585,853$ & 1685 \\
2016 & $527,803,014$ & 1689 \\
2017 & $528,999,628$ & 1693 \\
2018 & $530,116,356$ & 1696 \\
\hline
\end{tabular}

has a significant impact on prevalence. We recognise this model is based on a number of assumptions. The incidence rate is of primary importance in prevalence calculations, and can be difficult to determine in rare diseases. Recognition of a disease such as PMP may be delayed by a lack of knowledge and training of clinicians, leading to misdiagnoses and underestimation of incidence, even in countries with specialist centres. In contrast, the risk of overestimation of incidence is negligible.

The incidence rate used for our calculations was based on the number of people undergoing surgery for PMP in Norway and England. Those who were undiagnosed or not referred to the recording site would not be captured, so an incidence of 3.2 per million is probably an underestimate. Using the same waterfall plot calculation method, an incidence adjusted upward by just one per million per year to 4.2 would result in a prevalence of 15,130 people with
PMP living in Europe in 2018 whereas the previously assumed maximum incidence of 2 gives a prevalence of 7200 people. This illustrates the importance of establishing accurate incidence figures, including the provision of good quality central registries for rare disease surveillance.

The cure rate is also a key component of the prevalence calculation. A conservative cure rate of $47 \%$ was extrapolated from publications where all histological subtypes were included in countries where a curative treatment strategy is well established. The death rates per year were similarly derived from such publications. It is worth noting that the histological subtypes of PMP are associated with very different prognoses, potentially influencing both cure rate and death rate. A consensus for pathology classification of PMP was reached only relatively recently, ${ }^{19}$ and the broader implementation of these diagnostic principles is likely to improve the accuracy of reporting for more countries in the future.

Another influence on the prevalence is the availability of state-of-the-art treatment. In countries where specialist centres do not exist, treatment with curative intent is not likely to be available. This could, in principle, influence prevalence in two ways: either more patients may live with active disease for longer periods of time, leading to higher prevalence rates over time, or more patients may lose their lives earlier than would be expected, leading to lower prevalence rates. Similarly, the number of prevalent patients could be higher if these patients are offered repeat palliative surgical procedures, leading to longer overall survival, but with no concurrent change to their status as a patient under active care. Ultimately, one could speculate that these influences would balance each other out, but this remains an area of uncertainty. 
TABLE 4 Aggregative waterfall technique used to create the cascading prevalence chart using data from 2009 to 2018

\begin{tabular}{lrrrrrrrrrr}
\hline & \multicolumn{1}{c}{2009} & \multicolumn{1}{c}{2010} & \multicolumn{1}{c}{2011} & 2012 & 2013 & 2014 & 2015 & 2016 & 2017 & 2018 \\
\hline Total annual cohort & 1659 & 1664 & 1669 & 1673 & 1677 & 1681 & 1685 & 1689 & 1693 & 1696 \\
Cured (47\%) & $-\mathbf{7 8 0}$ & -782 & -784 & -786 & -788 & -790 & -792 & -794 & -796 & -797 \\
Year 1 $(-8 \%)$ & 1526 & 1531 & 1535 & 1539 & 1543 & 1547 & 1550 & 1554 & 1557 & 1561 \\
Year 2 (-14\%) & 1421 & 1426 & 1431 & 1435 & 1439 & 1442 & 1446 & 1449 & 1453 & 1456 \\
Year 3 (-20\%) & 1318 & 1322 & 1327 & 1331 & 1335 & 1339 & 1342 & 1345 & 1348 & 1351 \\
Year 4 (-22\%) & 1280 & 1285 & 1289 & 1294 & 1298 & 1302 & 1305 & 1308 & 1311 \\
Year 5 (-26\%) & 1209 & 1214 & 1219 & 1223 & 1227 & 1231 & 1235 & 1238 & 1241 \\
Year 6 (-29\%) & 1156 & 1160 & 1165 & 1169 & 1174 & 1178 & 1181 & 1185 & 1188 \\
Year 7 (-31\%) & 1119 & 1123 & 1128 & 1132 & 1136 & 1140 & 1144 & 1148 & 1151 \\
Year 8 (-33\%) & 1082 & 1086 & 1091 & 1095 & 1099 & 1103 & 1107 & 1111 & 1115 \\
Year 9 (-35\%) & 1046 & 1050 & 1054 & 1058 & 1062 & 1066 & 1070 & 1074 & 1078 \\
Year 10 (-37\%) & 1011 & 1014 & 1017 & 1021 & 1026 & 1030 & 1034 & 1038 & 1041 \\
Total period cohort & 12,168 & 12,212 & 12,256 & 12,298 & 12,339 & 12,378 & 12,415 & 12,450 & 12,484 & 12,516 \\
Minus 10-year survivors & 11,414 & 11,455 & 11,497 & 11,536 & 11,574 & 11,610 & 11,644 & 11,676 & 11,707 & $\mathbf{1 1 , 7 3 6}$ \\
\hline
\end{tabular}

Italics follow a single cohort through its iterative cycle; Bold numbers indicate the $47 \%$ rate of cure being applied to the full cohort in year 1 and removed from the cohort at the 10th year (total period cohort: accumulation of the annual incident cohorts over a 10-year period)

Incidence rates of "one in a million" seem attractive and imply a rarity that may not reflect reality. Often, understanding prevalence gives a more useful picture of the overall disease burden being experienced by society at large. This allows policy makers to establish effective strategies for diagnosis, treatment and palliation of rare conditions with centralisation of expertise and adequate funding as the NHS does in England through highly specialised commissioning for PMP. The responsibility for collecting accurate incidence data lies with healthcare professionals but needs support from policy makers so that, ultimately, patients benefit.

\section{CONCLUSION}

In this paper, we have presented a new method for determining the prevalence rates for rare diseases, using the slowly progressive cancer PMP as an example. The adjusted incidence rate of 3.2 people per million per year was based on epidemiological data from Norway and England. A prevalence rate of 22 people per million per year was calculated, which would mean that 11,736 people were living with PMP in Europe in 2018. Although PMP is a rare disease, given its slow and progressive nature, a significant number of people are living with the disease, putting pressure on European health-care systems. Standardising diagnosis and treatment pathways through education and training, sharing knowledge and expertise and establishing European research networks will optimise patients' outcomes. These prevalence calculations provide a starting point for identifying the burden of disease in this setting and may be a useful tool for similar estimates in other rare diseases. By investing in national and international registries, policy makers can validate these estimates to accurately inform healthcare strategy.

ACKNOWLEDGEMENT Open Access funding provided by University of Oslo (incl Oslo University Hospital). This article is based upon work from COST Action CA17101, the European Network on Pseudomyxoma Peritonei, supported by COST (the European Cooperation in Science and Technology).

DISCLOSURE The authors declare no competing interests.

OPEN ACCESS This article is licensed under a Creative Commons Attribution 4.0 International License, which permits use, sharing, adaptation, distribution and reproduction in any medium or format, as long as you give appropriate credit to the original author(s) and the source, provide a link to the Creative Commons licence, and indicate if changes were made. The images or other third party material in this article are included in the article's Creative Commons licence, unless indicated otherwise in a credit line to the material. If material is not included in the article's Creative Commons licence and your intended use is not permitted by statutory regulation or exceeds the permitted use, you will need to obtain permission directly from the copyright holder. To view a copy of this licence, visit http://creativecommons. org/licenses/by/4.0/.

\section{REFERENCES}

1. Chua TC, Moran BJ, Sugarbaker PH, et al. Early- and long-term outcome data of patients with Pseudomyxoma Peritonei from appendiceal origin treated by a strategy of cytoreductive surgery and hyperthermic intraperitoneal chemotherapy. J Clin Oncol. 2012;30(20):2449-56. 
2. Smeenk RM, van Velthuysen ML, Verwaal VJ, Zoetmulder FA. Appendiceal neoplasms and Pseudomyxoma peritonei: a population based study. Eur J Surg Oncol. 2008;34(2):196-201.

3. United Nations, Department of Economic and Social Affairs, Population Division. World Population Prospects 2019, Online Edition. Retrieved from https://population.un.org/wpp/Down load/Files/1_Indicators(Standard)/EXCEL_FILES/1_Population/ WPP2019_POP_F01_1_TOTAL_POPULATION_BOTH_SEXE S.xlsx. Accessed 26 July 2019.

4. United Nations, Department of Economic and Social Affairs, Population Division. Manual I: Methods for estimating total population for current dates. United Nations Publications, Sales No. 5.XIII.5. Retrieved from https://www.un.org/en/developme $\mathrm{nt} / \mathrm{des}$ /population/publications/manual/estimate/estimate-popula tion.asp. Accessed 26 July 2019.

5. Office for National Statistics. Analysis of population estimates tool. Retrieved from https://www.ons.gov.uk/peoplepopulationa ndcommunity/populationandmigration/populationestimates/datas ets/analysisofpopulationestimatestool. Accessed 11 December 2019.

6. Baratti D, Kusamura S, Nonaka D, Cabras AD, Laterza B, Deraco M. Pseudomyxoma peritonei: biological features are the dominant prognostic determinants after complete cytoreduction and hyperthermic intraperitoneal chemotherapy. Ann Surg. 2009;249(2):243-9.

7. Carr N, Finch J, Ilsley IC, et al.: Pathology and prognosis in pseudomyxoma peritonei: a review of 274 cases. J Clin Pathol. 2012;65(10):919-23.

8. Passot G, Vaudoyer D, Villeneuve L, et al. What made hyperthermic intraperitoneal chemotherapy an effective curative treatment for peritoneal surface malignancy: a 25-year experience with 1,125 procedures. J Surg Oncol. 2016;113(7):796-803.

9. Kitai T, Kawashima M, Yamanaka K, et al. Cytoreductive surgery with intraperitoneal chemotherapy to treat pseudomyxoma peritonei at nonspecialized hospitals. Surg Today. 2011;41(9):1219-23.

10. Bradley RF, Stewart JH, Russell GB, Levine EA, and Geisinger KR. Pseudomyxoma peritonei of appendiceal origin: a clinicopathologic analysis of 101 patients uniformly treated at a single institution, with literature review. Am $J$ Surg Pathol. 2006;30(5):551-9.

11. Youssef H, Newman C, Chandrakumaran K, Mohamed F, Cecil TD, Moran BJ. Operative findings, early complications and long- term survival in 456 patients with pseudomyxoma peritonei syndrome of appendiceal origin. Dis Colon Rectum. 2011;54(3):293-9.

12. Deraco M, Kusamura S, Laterza B, et al. Cytoreductive surgery and hyperthermic intra-peritoneal chemotherapy (HIPEC) in the treatment of pseudomyxoma peritonei: ten years experience in a single center. In Vivo. 2006;20(6A):773-6.

13. Elias D, Gilly F, Quenet F, et al. Pseudomyxoma peritonei: a French multicentric study of 301 patients treated with cytoreductive surgery and intraperitoneal chemotherapy. Eur J Surg Oncol. 2010;36(5):456-62.

14. Yan TD, Bijelic L, Sugarbaker PH. Critical analysis of treatment failure after complete cytoreductive surgery and perioperative intraperitoneal chemotherapy for peritoneal dissemination from appendiceal mucinous neoplasms. Ann Surg Oncol. 2007;14(8):2289-99.

15. Mercier E, Dagbert F, Pocard M, et al. Recurrence of pseudomyxoma peritonei after cytoreductive surgery and hyperthermic intraperitoneal chemotherapy. BJS Open. 2018;3(2):195-202.

16. Sørensen O, Flatmark K, Reed W, et al. Evaluation of complete cytoreductive surgery and two intraperitoneal chemotherapy techniques in pseudomyxoma peritonei. Eur J Surg Oncol. 2012;38(10):969-76.

17. van Eden WJ, Kok NFM, Snæbjornnsson P, et al. Factors influencing long-term survival after cytoreductive surgery and hyperthermic intraperitoneal chemotherapy for pseudomyxoma peritonei originating from appendiceal neoplasms. BJS Open. 2019;3(3):376-386.

18. Vaira M, Cioppa T, De Marco G, et al. Management of pseudomyxoma peritonei by cytoreduction + HIPEC (Hyperthermic Intraperitoneal Chemotherapy): results analysis of a twelve-year experience. In Vivo. 2009;23(4):639-44.

19. Carr NJ, Cecil TD, Mohamed F, et al. A consensus for classification and pathologic reporting of pseudomyxoma peritonei and associated appendiceal neoplasia: the results of the Peritoneal Surface Oncology Group International (PSOGI) modified Delphi process. Am J Surg Pathol. 2016;40(1):14-26.

Publisher's Note Springer Nature remains neutral with regard to jurisdictional claims in published maps and institutional affiliations. 